\title{
Auditory conditional discrimination deficits without delays in rats with lesions of either frontal cortex or medial thalamus
}

\author{
ALEXANDER A. STEVENS and ROBERT G. MAIR \\ University of New Hampshire, Durham, New Hampshire
}

\begin{abstract}
An auditory match-to-position (AMTP) task was developed to compare the effects of medial thalamic (L-IML) and prefrontal cortical (MW) lesions on the ability of rats to perform conditional discriminations with and without memory delays. Both lesions affected AMTP when there was no delay, and these impairments were not exacerbated when memory delays were imposed. The MW group performed normally, whereas the L-IML group was impaired in learning a go/no-go discrimination based on the same auditory stimuli as those used for AMTP. These findings are consistent with other evidence that the medial prefrontal cortex is critical for conditional associative learning when the same response alternatives are presented repeatedly across trials. The impairments observed for AMTP when there was no memory delay suggest that the effects of these lesions on the rate of forgetting may have been masked by the effects of these lesions on nonmnemonic aspects of the AMTP task.
\end{abstract}

There is controversy over whether amnesias, particularly those associated with thalamic lesions, impair memory by increasing the rate of forgetting or whether their main effects are on processes related to encoding or retrieval that might be expected to exert their effects on remembering in a manner that is independent of delay (Butters \& Cermak, 1980; Kopelman, 1992; Mayes, 1992; Parkin, 1987; Talland, 1965). Studies of nonhuman primate models have not settled this issue. Although several reports indicate that monkeys with lesions in the region of the mediodorsal thalamic nucleus (MDn) perform poorly on delayed conditional discrimination tasks (Aggleton \& Mishkin, 1983a, 1983b; Isseroff, Rosvold, Galkin, \& Goldman-Rakic, 1982; Zola-Morgan \& Squire, 1985), these studies have not compared performances for which retention intervals were varied randomly within sessions and, thus, do not provide an internally valid measure of forgetting (see AlvarezRoyo, Zola-Morgan, \& Squire, 1992).

In rats, large thalamic lesions affecting the MDn and the adjacent intralaminar nuclei (ILn) have been associated with impairments that are independent of the length of delay for delayed matching-to-sample (DMS) and de-

\footnotetext{
A preliminary report of this work has appeared in abstract form (Stevens \& Mair, 1995). This research constituted partial fulfillment of the degree requirements for a PhD at the University of New Hampshire for A.A.S. It was supported by NINDS Grant NS 26855 and by a Dissertation Fellowship from the University of New Hampshire. We thank Audrey Cantwell, Casey Carroll, Barbara Glode, and Virena Hermann for technical assistance and Clint Anderson, Steven Bornstein, Earl Hagstrom, and Tony Nevin for advice and criticism. A.A.S. is now a postdoctoral associate in the Department of Diagnostic Radiology at Yale University School of Medicine. Correspondence concerning this article should be addressed to R. G. Mair, Psychology Department, University of New Hampshire, Durham, NH 03824 (e-mail: rgm@) christa.unh.edu).
}

layed nonmatching-to-sample (DNMS) tasks using spatial location (Mair, Burk, \& Porter, 1998; Robinson \& Mair, 1992; Young, Stevens, Converse, \& Mair, 1996), trial-unique objects (Mumby, Mana, Pinel, David, \& Banks, 1995; Mumby, Pinel, \& Dastur, 1993), and odorants (Koger \& Mair, 1994; Zhang, Burk, Glode, \& Mair, $1998)$ as discriminative stimuli. Similar impairments have been reported for rats with thalamic lesions produced by pyrithiamine-induced thiamin deficiency (PTD), by radiofrequency (RF) current that damaged lateral portions of the internal medullary lamina (L-IML), or by $N$-methyl$\mathrm{D}$-aspartate (NMDA) infusions aimed at the ILn.

There are several reasons to think that some of the effects of thalamic lesions on remembering can be explained by their impact on the prefrontal cortex (PFC). The PFC is innervated by the MDn and the ILn and has been shown to be denervated by both PTD treatment and RF lesions of the L-IML site (Mair, Ferguson, Knoth, \& Langlais, 1989; Zhang \& Mair, 1992). Other studies have shown that lesions of the PFC along the medial wall (MW) of the cerebral hemispheres produce behavioral impairments comparable with those due to thalamic lesions. Thus, RF lesions of the MW area affect both the speed and the accuracy of responding on DMS and DNMS tasks using adjacent response ports, alleyways, or retractable levers as discriminative stimuli-disrupting response accuracy in a delay-independent fashion and increasing the frequency of long-latency responses (Mair et al., 1998; Porter \& Mair, 1997; Young et al., 1996). Comparable impairments have been observed on these same tasks following PTD treatment (Robinson \& Mair, 1992), RF L-IML lesions (Young et al., 1996), and NMDA ILn lesions (Burk \& Mair, 1998; Mair et al., 1998). For other measures of remembering, PFC lesions have relatively minor effects, as compared with those of ILn or 
L-IML lesions (Mair et al., 1998). Thus, the involvement of the PFC does not seem adequately to account for the full range of impairment associated with these thalamic lesions.

The behavioral deficits associated with PTD, L-IML, and ILn thalamic lesions have been taken as evidence of a working memory impairment, on the basis of findings that lesioned animals retain the ability to learn and to perform simple sensory discriminations that do not require rats to remember stimulus information on a trial-by-trial basis (Mair, 1994; Mair et al., 1998). Similar dissociations have been found for rats with PFC lesions (Harrison \& Mair, 1996; Koger \& Mair, 1994; Mair et al., 1998). Although simple discriminations can control for a number of the procedural and sensory demands of DMS or DNMS tasks, they do not control for at least one important nonmnemonic factor: the conditional choice between discriminative stimuli and the behavior they occasion. There are at least two reasons to suspect that medial thalamic lesions affect the ability to perform conditional discriminations. First, such an explanation is consistent with the evidence that thalamic lesions disrupt DMS and DNMS performance when delays are minimized and that the severity of these deficits is not exacerbated when the delays are lengthened. Thus, there seems to be no compelling reason to conclude that these DMS and DNMS impairments result from an inability to remember stimulus information rather than an inability to perform conditional discriminations. Second, Savage and Langlais (1995) have reported that the effects of PTD treatment on a place DMS task are attenuated significantly when rats are trained with a differential outcomes procedure. It is well known that conditional discrimination performance is enhanced by differential outcomes training with qualitatively different reinforcers (Trapold, 1970). Thus, although the DMS performances of PTD-treated rats are unchanged by manipulations of retention interval that are predicted to affect mnemonic difficulty, they appear to be attenuated by differential outcomes training, which is predicted to lessen the difficulty of the conditional discrimination.

One source of uncertainty that limits the interpretation of delayed conditional discrimination deficits is the lack of data allowing direct comparison of conditional discrimination performances with and without memory delays. Many previous studies have relied on spatial locations as discriminative stimuli for DMS or DNMS tasks. Unfortunately, such stimuli require that the animals physically disengage from the sample location before making a choice response and, thus, impose a limit on the minimal delay that can be tested. The use of nonrecurring objects or of odorants as stimuli also places limits on the minimal delays that can be studied. To avoid this limitation, we trained rats to perform an auditory match-to-position (AMTP) task in which two distinct tones were associated with the availability of reinforcement in two different spatial locations. The use of tones provided precise control over temporal aspects of the discriminative stimulus that could be presented or removed at any point during the conditional response task. In addition, the rats were tested on a go/no-go version of the AMTP task, to determine to what degree response requirements determined performance. Two experimental treatments were studied, each of which has been associated with delay-independent memory impairments: RF lesions of the L-IML site in the thalamus and RF lesions of the medial wall (MW) area of the frontal cortex. The L-IML lesion has been found to impair performance in spatial DNMS and DMS (Mair \& Lacourse, 1992; Young et al., 1996), in continuous olfactory DNMS (Koger \& Mair, 1994), and on an eight-arm radial maze with imposed delays (Harrison \& Mair, 1996). The MW area of the frontal cortex is denervated by the L-IML lesion (Zhang \& Mair, 1992). Lesions of this area have been found to impair place DNMS and DMS (Mair et al., 1998; Porter \& Mair, 1997; Young et al., 1996), while sparing olfactory DNMS (Koger \& Mair, 1994) and some versions of DNMS trained in the radial-arm maze (Porter \& Mair, 1997).

\section{METHOD}

\section{Subjects}

Thirty male Long-Evans rats began training at 6 weeks of age (Charles River Laboratories). The rats were housed individually in stainless steel cages and maintained on a 12:12-h light:dark cycle, with training taking place during the light phase. They were given ad-lib access to food and were maintained on a schedule of $23 \mathrm{~h}$ of water deprivation in order to make water a salient reinforcer. On days when they were trained, rats received water $(7-10 \mathrm{ml})$ during training sessions and for 30 min per day at the start of the dark phase of the light:dark cycle.

\section{Treatment}

The rats were assigned to treatment groups, using a randomized matching procedure based on percent correct performance during the last three sessions of presurgical training. The rats were grouped into blocks of 3 according to this measure, and 1 in each block was randomly assigned to each of the three treatments. Lesions were made following surgical procedures like those described by Young et al. (1996) for making RF lesions of the L-IML and MW sites. The rats were given ad-lib water for $48 \mathrm{~h}$, and food was removed for $12 \mathrm{~h}$ prior to surgery. The rats were anesthetized by IM injections of xylazine $(8.5 \mathrm{mg} / \mathrm{kg})$ and ketamine $(85 \mathrm{mg} / \mathrm{kg})$ and placed in a Kopf stereotaxic instrument with the incisor bar $3.3 \mathrm{~mm}$ below the interaural line (IA). The skull overlying the lesion site was opened, using sterile techniques and lesions made by lowering a Radionics TCZ probe to the appropriate coordinates, passing RF current, and monitoring tip temperature with a Radionics RFG-4A lesion generator. The rats in the sham group were treated like the lesion groups, with the exception that the skull was not opened and the electrode was not lowered into the brain tissue.

L-IML lesions were made by heating the electrode tip to $70^{\circ} \mathrm{C}$ for $30 \mathrm{sec}$ at each of 12 locations, bilaterally $1.0 \mathrm{~mm}$ off the midline (ML) at each of two depths (dorsal-ventral 3.8 and 4.8 from IA) at three anterior posterior (AP) levels $(5.2,6.2$, and $7.2 \mathrm{~mm}$ from IA). The much larger $\mathrm{MW}$ lesions were made by heating the electrode tip to $75^{\circ} \mathrm{C}$ for $30 \mathrm{sec}$ at sites located at five AP levels $(4.7,3.7,2.7$, 1.7 , and $0.7 \mathrm{~mm}$ in front of bregma). At each of these AP levels, bilateral lesions were made $0.8 \mathrm{~mm}$ from the $\mathrm{ML}$ at 1.0 and $2.2 \mathrm{~mm}$ beneath the surface of cortex, except at $\mathrm{AP}=4.7$, where the more ventral lesion was omitted. More lateral lesions were also made at the three most anterior levels $(2.7,3.7$, and $4.7 \mathrm{~mm}$ from bregma) at $2.0 \mathrm{~mm}$ from ML, $1.0 \mathrm{~mm}$ beneath the surface of the cortex. 


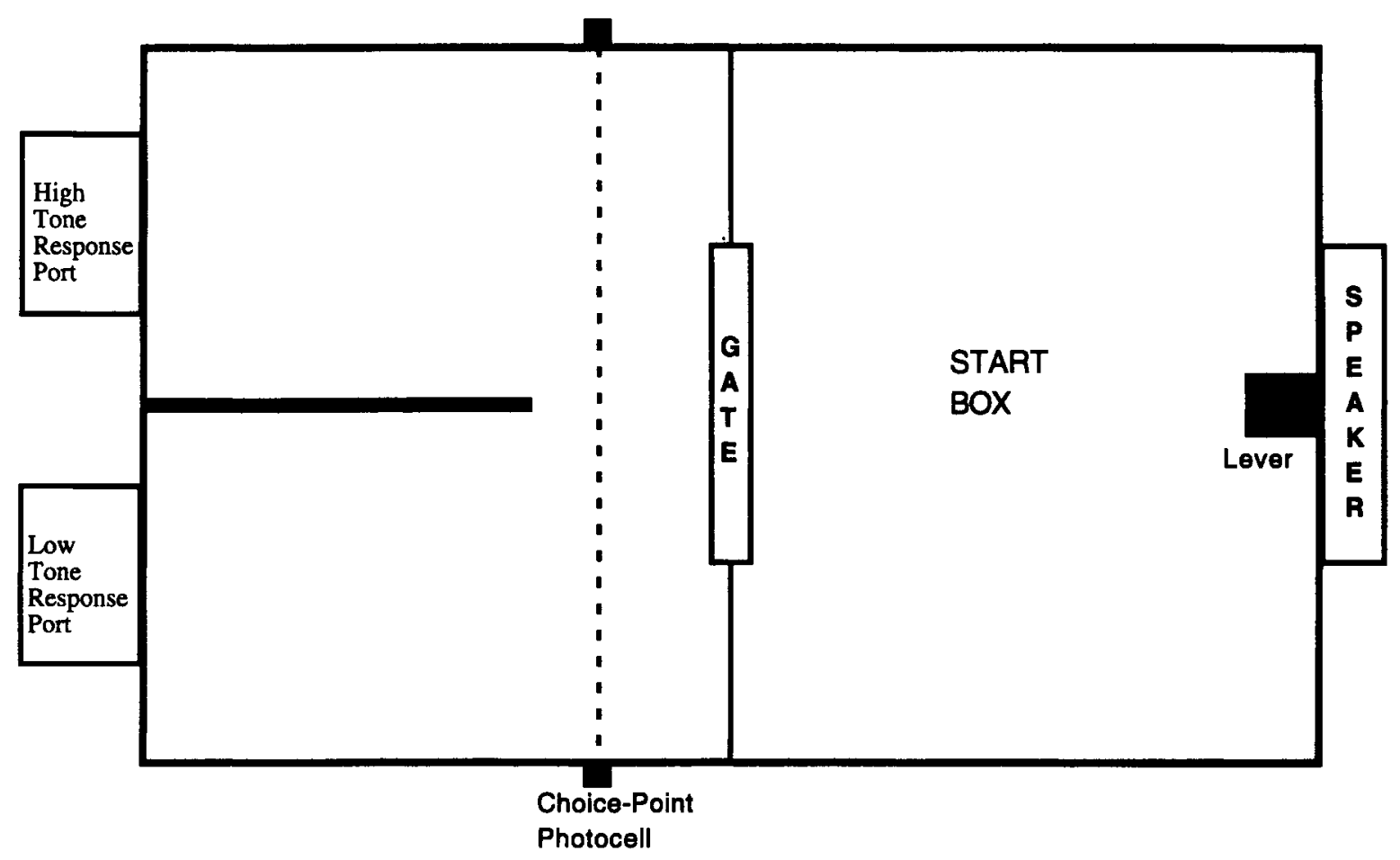

Figure 1. Schematic of the apparatus used for the auditory match-to-position (AMTP) task and the go/no-go discrimination task. All the trials began with the animal in the startbox and the gate closed. The auditory stimulus was presented for 2 sec before the gate was lifted. For AMTP, the tones were turned off at different times on different types of trials: after a discriminative response was made on discrimination trials, when the photobeam at the choice point was broken on choice point trials, or at delays of 0, 0.4, 1.0, or 2.5 sec before the gate was raised on retention interval trials. During the go/no-go task, a barrier blocked access to the high-tone response port.

\section{Apparatus}

Three test chambers were used, each constructed by joining two Lafayette 85000 Modular Units, separated by a motorized stainless steel guillotine door (Figure 1). The modular units measured $31 \times$ $18 \times 20 \mathrm{~cm}$, with a floor of stainless steel bars $(0.4 \mathrm{~cm}$ in diameter spaced $1.7 \mathrm{~cm}$ apart, center to center). The ceiling was translucent Plexiglas that was illuminated by a 28 -W houselight. There was a pan of clean pine shavings beneath the floor. One of the modular units was used as the startbox and was equipped with a response lever set $4.5 \mathrm{~cm}$ above the floor in the center of the wall opposite the door and with a speaker (ENV-224, MED Associates, Georgia, VT) directly above the lever. The other modular unit served as the choice box and was equipped with two response ports, constructed from PVC tubing ( $5 \mathrm{~cm}$ in diameter), positioned $3 \mathrm{~cm}$ above the floor and centered $10.5 \mathrm{~cm}$ apart, center to center, on the wall opposite the guillotine door. The response ports were separated by an opaque Plexiglas divider $17.5 \mathrm{~cm}$ high and extending $12 \mathrm{~cm}$ into the choice box. To make the two response ports more distinct, the wall adjacent to the left response port was painted black and a small 2-W light was positioned above the right response port. Each response port had a stainless steel drinking spout, through which reinforcements $(0.1 \mathrm{ml}$ of tap water) were delivered by brief activation of a miniature solenoid valve (LFAA 1201518 H, The Lee Co., Essex, CT), a photocell positioned just in front of the drinking spout to detect responses, and a $5-\mathrm{W}$ signal light positioned at the back of the port. A third photocell was mounted in the choice box, oriented across the chamber $6 \mathrm{~cm}$ from the guillotine door $(2 \mathrm{~cm}$ in front of the divider) to detect a rat's arrival at the choice point.

Two tones $(2 \mathrm{kHz}$ and $11 \mathrm{kHz})$ served as stimuli and were generated by a solid state programmable acoustic stimulator (MED As- sociates) and presented at a sound pressure level of $85 \mathrm{~dB}$ (Ged Rad Scale $C$ measured at $3 \mathrm{~cm}$ from the speaker). The acoustic stimulators and the training chambers were controlled by a 486 personal computer via a commercially available interface (MED Associates).

\section{Procedure}

Presurgical training. During initial shaping, the rats learned to press the startbox lever to produce a continuous low $(2-\mathrm{kHz})$ tone and then to lick the drinking tube in the left response port to obtain a reinforcer. Training was conducted in daily sessions of 100 trials until rats reached a criterion of 2 consecutive days in which they responded to the left (correct) response port before responding to the right port on $90 \%$ of the trials. We then modified the procedure so that the gate was closed following the first leverpress of a trial and the 2-kHz tone turned on following the second press. Two sec after the tone was turned on, the gate was re-opened, allowing the animal access to the choice box where reinforcement was given, following a response to the left port. Training was carried out in daily sessions of 100 trials, with a $3-\mathrm{sec}$ intertrial interval (ITI) until rats achieved a level of $90 \%$ correct for two consecutive sessions.

We then began AMTP training by introducing a high $(11-\mathrm{kHz})$ tone on $50 \%$ of the trials, during which reinforcement was given for licking the drinking spout on the right (illuminated) side of the choice box. High- and low-tone trials were presented in a randomized order, with the constraint that neither stimulus was repeated on more than 4 consecutive trials. Responses were scored as correct when the rats responded first to the correct port (left, black side for the low tone; right, illuminated side for the high tone). During initial AMTP sessions, we used a correction procedure in which trials continued after an incorrect response until a reinforcement was eventually obtained 
for a response to the correct side. This correction procedure was discontinued after individual animals reached a level of $70 \%$ correct responding. This version of AMTP was considered a discrimination task, because the tone remained on until the response was made and, thus, there was no requirement that the tone be remembered during a delay period. Training continued on the AMTP discrimination task until animals performed at least $85 \%$ correct for 2 consecutive 100 trial sessions or for a maximum of 3,000 trials ( 30 sessions).

Postsurgical AMTP. After 10-12 days of recovery from surgery, the rats received five consecutive phases of AMTP training. During the initial postsurgical phase, the rats were trained ( 60 trials/session) with the discrimination version of AMTP that was used prior to surgery. The mnemonic demands of the AMTP task was varied during the training phases by varying the retention intervals imposed. To facilitate postsurgical reacquisition of AMTP, we trained the rats with the correction procedure on an alternate session-bysession basis during the first 5 sessions. After this, the correction procedure was used for individual animals on an alternate sessionby-session basis when performance fell below $70 \%$ accuracy (during the initial version of AMTP only). Data from the sessions in which the correction procedure was used were excluded from analyses of postsurgical performance. The subjects received a minimum of 900 trials ( 15 sessions without the correction procedure). Training continued until rats averaged $85 \%$ correct or better for 3 of 5 consecutive sessions or until rats completed a maximum of 1,800 trials ( 30 sessions).

In the second phase, discrimination trials were intermixed with choice point trials in which the tones turned off after rats entered the choice box and broke the photocell beam in front of the partition that separated the response ports (Figure 1). Thus, the tones were kept on up to the point at which the rats had to enter one of the two response ports. The rats were trained with this version of AMTP for 10 sessions, each consisting of 30 discrimination and 30 choice point trials. The order of the different trial types was randomized, with the constraints that no more than 2 trials of one type (discrimination vs. choice point) or no more than 4 trials with the same stimulus (high vs. low tone) could occur in a row.

In the third phase, we trained the rats for 20 sessions in which the discrimination and choice point trials were intermixed with trials in which the tone was turned off still earlier-when the gate was raised to allow animals access to the choice box. Since the lifting of the gate marked the first time at which animals could see the response alternatives, this was considered to be a 0 -sec retention interval (RI) and, thus, these were called RI $=0$ trials. Each session consisted of 60 trials, evenly divided in a randomized sequence of the three trial types, subject to the constraint that no more that 2 trials of one type or no more than 4 trials with the same stimulus could occur consecutively.

In the fourth phase of training, we increased the mnemonic demands of AMTP by lengthening the RI from 0 to $2.5 \mathrm{sec}$. There were five types of trials, including choice point trials (as described above) and trials in which tones were turned off at $0,0.4,1.0$, and $2.5 \mathrm{sec}$ prior to the gate opening (termed $\mathrm{RI}=0,0.4,1.0$, and 2.5 trials). The choice point trials were included to maintain the association between stimuli and their corresponding response ports. These were trained in blocks of 5 trials at the start and after the 15th, 30 th, and 45 th trials of each session. Each session consisted of 20 choice point trials and 10 trials at each of the four different RIs. The four RI conditions were tested in blocks of 10 trials in an order that was counterbalanced across the 16 sessions in which this version of AMTP was trained.

In the last phase of AMTP training, we tested the extent of postsurgical recovery by training animals for 10 sessions with the same discrimination procedure as that followed during the presurgical and the initial postsurgical training.

Auditory go/no-go discrimination. To further clarify the nature of the behavioral impairments, we next trained the rats to perform a discrimination that resembled AMTP, except that the be- havioral response was changed to a go/no-go procedure, rather than a spatially defined conditional discrimination. The go/no-go task used the same test chambers as those for AMTP, with the modification that the right response port was blocked off, leaving access only to the left response port, and the gates were kept open at all times. The high $(11-\mathrm{kHz})$ tone served as the $\mathrm{S}-$ and the low $(2-\mathrm{kHz})$ tone as the $\mathbf{S}+$ stimuli, signaling the availability of reinforcement on a given trial.

The subjects were initially trained in sessions of all $\mathrm{S}+(2-\mathrm{kHz})$ trials. As in AMTP, trials were initiated by a leverpress in the startbox. Reinforcers were delivered when the rats responded within a response window that was reduced from 20 to $7 \mathrm{sec}$ over 5 to 10 sessions. The stimulus tone remained on throughout the response window or until a response was made. When the rat completed 2 sessions of 100 trials with fewer than five misses, the $\mathrm{S}-$ trials were introduced, signaled by an $11-\mathrm{kHz}$ tone. On $\mathrm{S}-$ trials, licks at the water spout in the response port were not reinforced and were counted as a false alarm (FA). Trials terminated after $7 \mathrm{sec}$. In addition, the rat could terminate any trial by completing a fixed interval 2-sec (FI-2) response schedule on the lever following the onset of the tone. Responses were scored as follows: On S+ trials, a response to the response port within $7 \mathrm{sec}$ was scored as a correct response (hit), whereas failures to respond within $7 \mathrm{sec}$ or completion of the FI-2 schedule on the lever were counted as errors. On the Strials, a response to the choice port within $7 \mathrm{sec}$ was treated as an FA. Withholding a response for $7 \mathrm{sec}$ or completion of the FI-2 schedule on the lever was counted as a correct rejection (CR). A CR was always followed by an $\mathrm{S}+$ trial, but this trial was not used in the calculation of response accuracy. Trial types were presented in a pseudorandom order such that neither $S+$ nor $S$ - trials could occur more than four times in succession. All the trials were followed by a 3 -sec ITI. FA responses were followed by up to 2 correction trials, which were identical to the $S$ - trials but were not included in the analyses. The session returned to the pseudorandom schedule following a $\mathrm{CR}$ on a correction trial or after correction trials. The rats were trained for 15 sessions of 60 trials.

Histological processing. After completion of the go/no-go training, the rats with experimental lesions were sacrificed under deep anesthesia $(100 \mathrm{mg} / \mathrm{kg}$ ketamine, $10 \mathrm{mg} / \mathrm{kg}$ xylazine IM) by transcardiac perfusion of physiological saline, followed by $5 \%(\mathrm{v} / \mathrm{v})$ neutral buffered formalin. Brains were cyroprotected by subsequent immersion in solutions of $10 \%$ glycerin $/ 4 \%$ neutral buffered formalin and then $20 \%$ glycerin $/ 4 \%$ neutral buffered formalin. Tissue was sectioned frozen in the coronal plane at $30 \mu \mathrm{m}$. Every fifth section was then mounted and stained with cresyl violet for histological verification of the extent of the lesions.

\section{RESULTS}

\section{Histological Analyses}

The criteria for the L-IML lesion were: AP from the anterior thalamic nuclei to the fasciculus retroflexus, DV from the habenula to the depth of the ventral margin of the central medial nucleus, and ML bilaterally symmetric centered from 0.9 to $1.1 \mathrm{~mm}$ from the ML. Data from the $1 \mathrm{~L}-\mathrm{IML}$ animal that did not meet these criteria were eliminated from the analyses of behavioral performance. The criteria for the MW lesion were complete or near complete destruction of areas FR2, CG1, and CG3 along the shoulder and medial wall of the PFC throughout its anterior-posterior extent, as defined by Zilles (1985). All the rats in the MW group had criterion lesions and were thus included in subsequent analyses of behavioral data. Figure 2 illustrates the largest and smallest lesions meeting the criteria for the two treatment groups. 


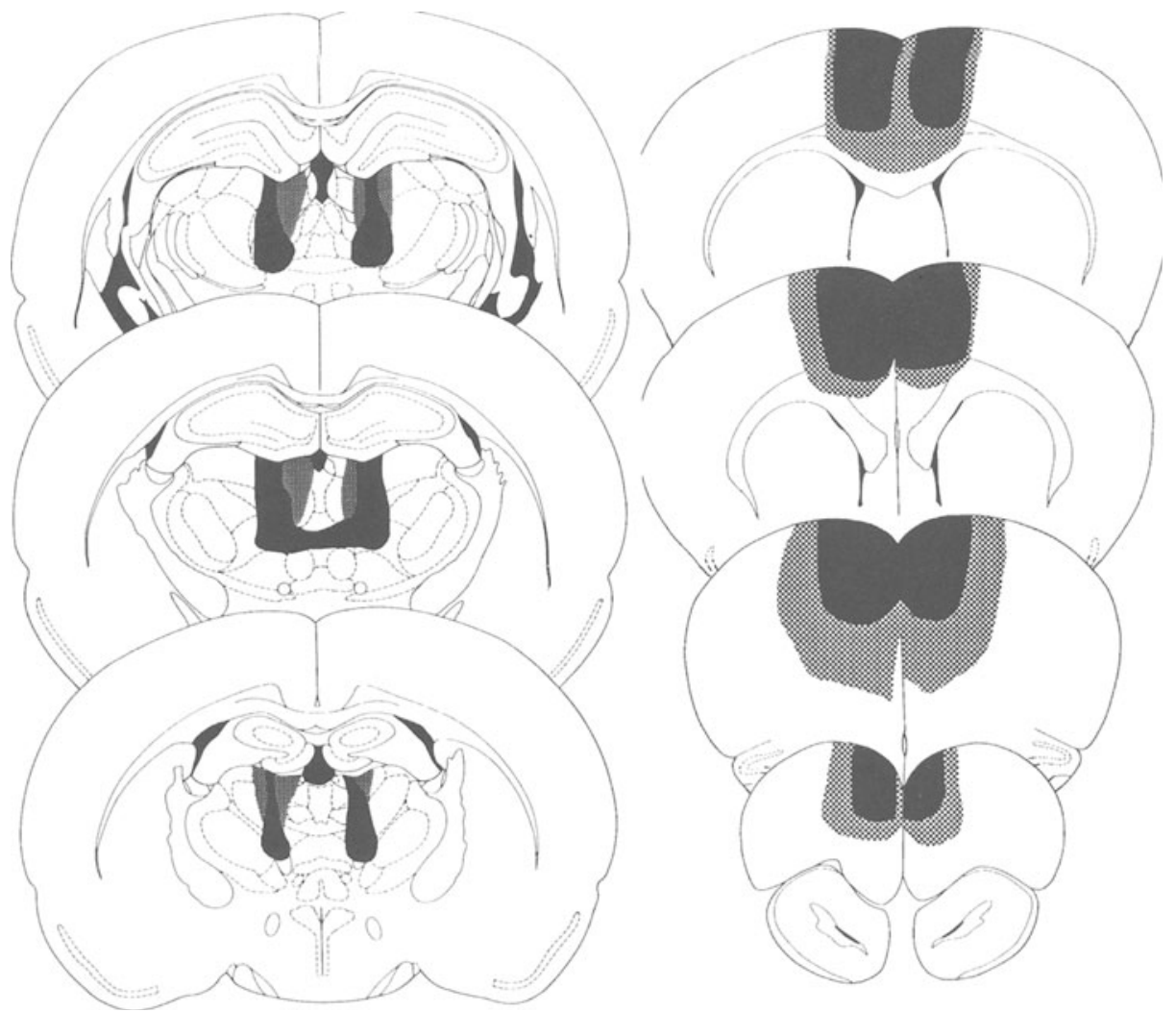

Figure 2. Extent of the largest (stippled) and smallest (black) thalamic (L-IML) lesions and prefrontal cortical medial wall (MW) lesions. Results are shown on the left for the L-IML lesions at 5.2, 6.2, and $7.2 \mathrm{~mm}$ anterior to the interaural (IA) line. The MW lesions are shown on the right at 9.2, 10.7, 12.2, and $13.7 \mathrm{~mm}$ anterior to the IA. Templates are modified from Paxinos and Watson (1986).

\section{Behavioral Performance}

AMTP. During the last 300 trials of presurgical training, rats assigned to the control, L-IML, and MW groups performed equivalently, averaging $81.5 \%, 82.4 \%$, and $81.7 \%$ correct, respectively (see Figure 3 ). These differences were not statistically significant when compared by a one-factor analysis of variance (ANOVA; $F<1$ ).

Following recovery from surgery, all the animals were retested for a minimum of 900 trials on the AMTP discrimination procedure in which tones were present continuously until a response was made (Phase 1). The rats continued on this procedure until they reached criterion of 3 of 5 sessions above $85 \%$ or 1,800 completed trials. All animals $(8 / 8)$ in the control group met the $85 \%$ criterion within 900 trials, whereas only $1 / 7$ of the L-IML animals reached this criterion over 1,800 trials. The performance of the MW group was intermediate between the control and the L-IML groups. Only $1 \mathrm{MW}$ animal reached criterion within the first 900 trials, but $7 / 8$ did so within 1,800 trials. When performances were compared over each animal's last 600 trials ( 10 sessions) of Phase 1 , the control group averaged $84 \%$, whereas the L-IML group performed at $71 \%$ accuracy and the MW group reached $77 \%$ correct. An ANOVA showed that the performances of the groups were significantly different $[F(2,20)=$
$12.033, p=.0004]$. Post hoc analyses (Student-NewmanKeuls, $\alpha=.05$ ) indicated that both the MW and the LIML groups were impaired, as compared with the control group, and that the L-IML group was impaired, as compared with the MW group.

In Phase 2, the MW and L-IML groups were both impaired for the discrimination trial and for the choice point trials in which tones were turned off as soon as the rats reached the partition separating the two response alternatives. Comparison of the two types of trials showed that turning tones off at the choice point had little effect on performance (Figure 4). These trends were verified by a two-factor (treatment $\times$ trial type) ANOVA showing a significant effect of treatment $[F(2,20)=5.374, p=.0136]$ but no effect of trial type $[F(1,20)=1.074$, n.s. $]$ and no interaction between treatment and trial type $[F(2,20)=$ 1.110 , n.s.]. Post hoc analyses (Student-Newman-Keuls, $\alpha=.05$ ) indicated that the difference between the controls and the L-IML group was significant but that the MW animals did not differ significantly from either the L-IML or the control group.

In Phase 3, discrimination and choice point trials were intermixed with $\mathrm{RI}=0$ trials in which the tone was turned off as soon as the gate was raised to allow access to the chamber containing the two response ports. All three 


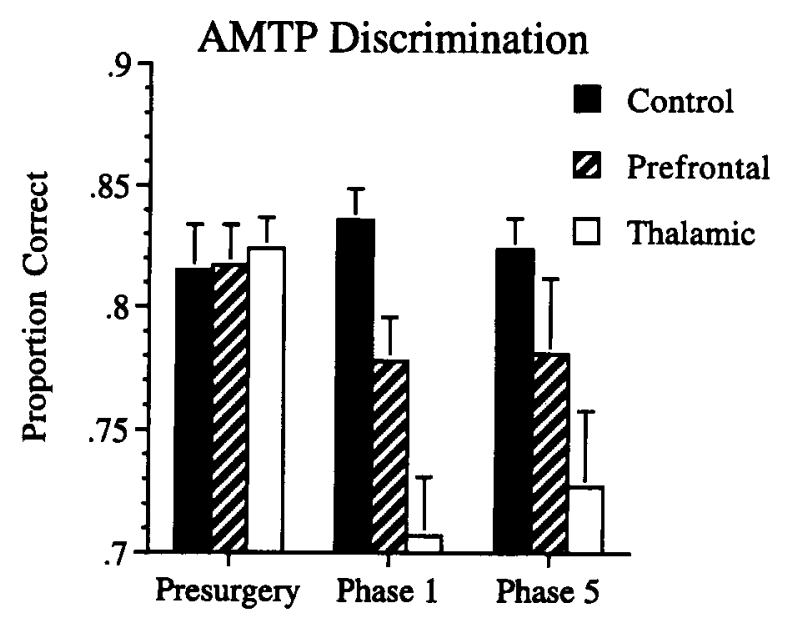

Figure 3. Proportion correct on discrimination trials during presurgical training and Phases 1 and 5 of postsurgical training. Results are shown for the control, prefrontal medial wall, and thalamic (L-IML) lesion groups. On discrimination trials, the tone remained on until a response was made to one of the response ports and, thus, there was no requirement for it to be remembered. Presurgical data include the final 300 trials for each rat included in the study. Phase 1 data include the final 600 continuous tone trials for each animal during the first phase of testing. The Phase 5 data followed a minimum of 4,500 trials for each rat. It includes all 600 discrimination trials for each animal.

groups were much less accurate on these trials. Although the relative rankings of the three groups were the same for all three trial types, the group differences were compressed by poor performance on the RI $=0$ trials (Figure 5 ). Statistical analyses were based on the final 10 (of 20) sessions, when performances had stabilized after initial inconsistency. In these analyses, treatment effect was only marginally significant $[F(2,20)=3.085, p=.0680]$, presumably because of the compression of performances during the $\mathrm{RI}=0$ trials. The poor performance during the $\mathrm{RI}=0$ trials was confirmed by the significant effect of trial type $[F(2,40)=130.214, p<.0001]$. The interaction between trial type and treatment was not statistically significant $[F(4,40)=1.667, p=.1766]$.

When RIs were increased up to $2.5 \mathrm{sec}$ during Phase 4 of training, all three groups performed less accurately at longer RIs. As can be seen in Figure 6, the forgetting functions for the three groups were parallel to each other, with the MW group exhibiting a level of impairment intermediate between the L-IML and the control groups. These trends were verified by a two-factor (treatment $X$ RI) ANOVA demonstrating a significant effect of treatment $[F(2,19)=4.34, p<.028]$, a significant effect of $\mathrm{RI}[F(3,57)=13.93, p<.0001]$, but no interaction between these factors $(F<1)$. Post hoc analyses (StudentNewman-Keuls, $\alpha=.05$ ) demonstrated that the L-IML group was impaired, as compared with the control and MW groups, but that the latter two groups did not differ from each other. It should also be noted that the extent of the treatment effects observed during the choice point trials (plotted along the ordinate in Figure 6 but not in- cluded in the RI analyses) was at least as large as during any of the delay trials.

In Phase 5, the extent of postsurgical recovery was assessed by retraining rats on the discrimination task run during the first phase of postsurgical training. Although the performance of the L-IML group was improved from Phase 1, they remained impaired compared with the control and the MW groups (Figure 3). An ANOVA showed that the differences between groups in this phase of training was only marginally significant $[F(2,20)=3.392, p=$ .054 ].

Go/no-go discrimination. Both the control and the MW groups learned the go/no-go discrimination rapidly until their performances stabilized between the 5th and the 10th sessions (Figure 7). The L-IML group performed much more poorly, averaging $69 \%$ correct across their last 5 sessions, as compared with $95 \%$ for the controls and $93 \%$ for the MW group. A one-way ANOVA on treatment effect indicated that these differences were significant $[F(2,20)=11.397, p<.0005]$. Post hoc analyses (Student-Newman-Keuls, $\alpha=.05$ ) demonstrated that the L-IML group was impaired, as compared with the control and MW groups, but that these latter two groups did not differ from each other.

\section{DISCUSSION}

The results of this study demonstrate that both the LIML and the MW lesions affected AMTP performance during discrimination trials, in which the tone was kept on until a response was made and in which, thus, there was no requirement to remember information across time. Turning the tone off earlier, just prior to when the rats entered one of the two alleyways in the choice box, had little effect on

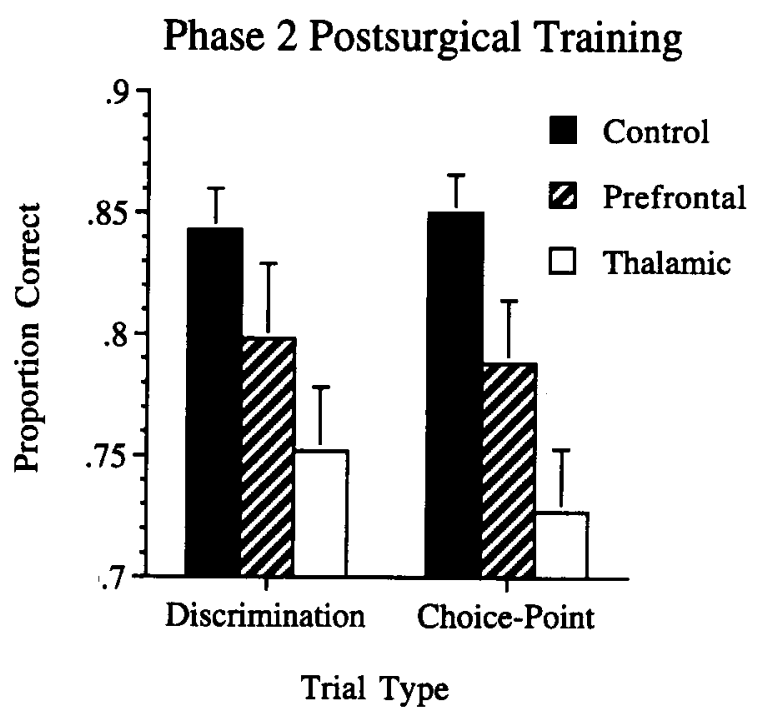

Figure 4. Proportion correct for the control, prefrontal, and thalamic lesion groups for the $\mathbf{3 0 0}$ discrimination trials and $\mathbf{3 0 0}$ choice point trials run during Phase 2 of postsurgical training. During choice point trials, the tone terminated when the rat broke the photobeam in front of the divider in the response box. 


\section{Phase 3 Postsurgical Training}

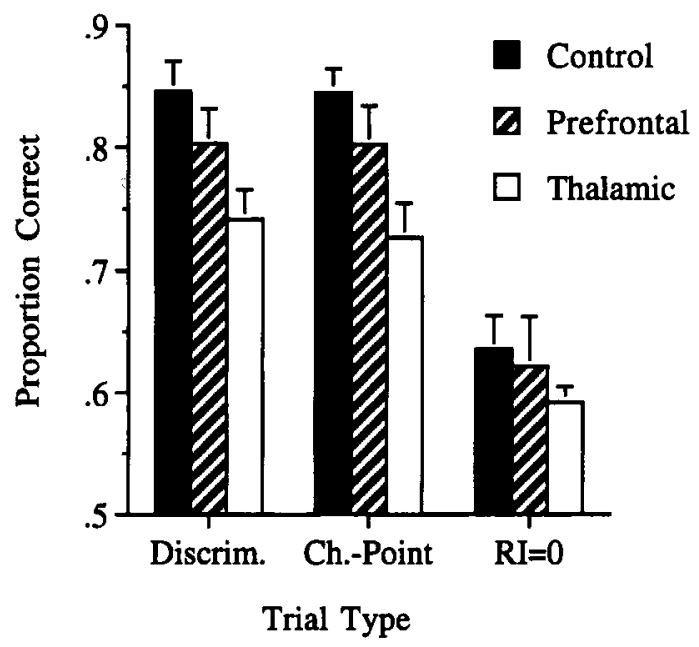

Figure 5. Proportion correct for the control, prefrontal, and thalamic lesion groups during Phase 3 of postsurgical training. Results are averaged across the $\mathbf{4 0 0}$ trials of the discrimination, choice point, and $R I=0$ trial types. On the $R I=0$ trials, the tone terminated with the raising of the gate.

performance for any of the groups, although post hoc tests did not show the MW group to be significantly impaired during the second phase of training, when these trials were introduced. Increasing mnemonic demands by turning the tone off at longer delays, before rats were allowed to enter the choice box, had significant effects on performance; however, this manipulation did not exacerbate the impairments associated with either the thalamic or the prefrontal cortical lesion. Both the impairment observed during discrimination trials, in which there was no delay, and the finding that the degree of impairment was independent of the length of the retention interval when delays were imposed provide evidence that the effects of these lesions on AMTP do not depend on the mnemonic requirements of this task.

There are, however, two important reasons to be cautious about this interpretation, particularly for the L-IML group. First, the lesioned animals performed so poorly during the discrimination trials that the effects of subsequent delay manipulations were almost certainly limited by a floor effect. Second, the poor performance of the LIML group on the go/no-go task is indicative of a more general inability to respond differentially to the two pure tone stimuli. Such an impairment may have masked any effects of the L-IML lesion on the ability to remember tones in the AMTP task.

\section{Effects of Prefrontal Cortical Lesions}

The MW group was impaired for AMTP but not for the go/no-go discrimination. The AMTP impairment was statistically significant during Phase I of training, during which there was no memory delay. Although the effects of the MW lesion were not statistically significant during other phases of training, the deficits observed for discrimination and choice point trials showed little change throughout postsurgical training (compare Figures 3, 4, 5 , and 6). The effects of this lesion were reduced as overall group differences were compressed by an apparent floor effect when temporal delays were imposed during later phases of training. The spared performance of the go/no-go task indicates that auditory function was intact and that animals with MW lesions were able to learn to use tones to mediate differential responses for the go/nogo discrimination. The impairment of AMTP is indicative of an inability to perform a simultaneous two-choice conditional discrimination based on the same auditory stimuli. It seems unlikely that this impairment was related to difficulty remembering information about the discriminative stimuli, given the findings that AMTP was impaired when there was no memory delay imposed and the impairment was not exacerbated when delays were imposed and increased in length. Thus, it appears that the effects of the MW lesion are related to the response requirements of the AMTP task and not to the difficulty of either discriminating or remembering the tones that served as discriminative stimuli.

The most apparent difference between AMTP and the go/no-go task is in the nature of the discriminative response. In AMTP, the rats were reinforced repeatedly in two different locations and needed to learn to select one side or the other on the basis of the pitch of the discriminative stimulus. Thus, through differential reinforcement, the rats were required to learn to associate discrete tones with discrete locations of reinforcement. In the go/no-go task, reinforcement was given in only one location, and,

\section{Phase 4 Postsurgical Training}

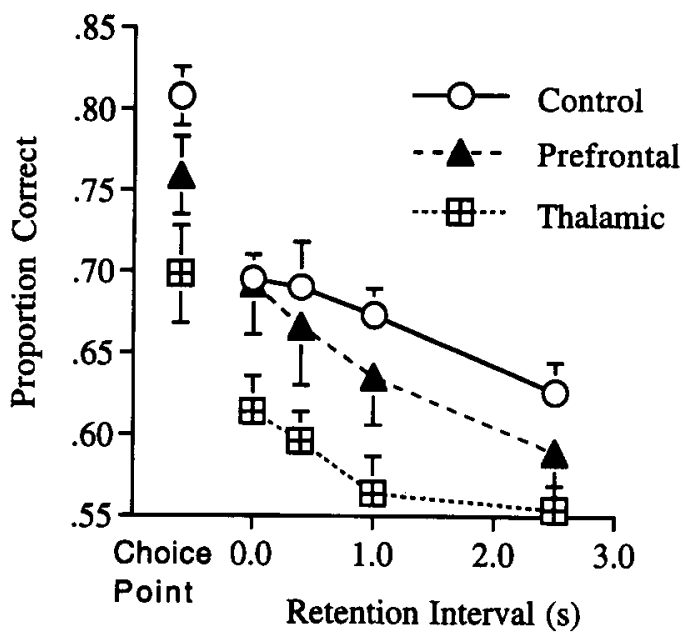

Figure 6. Proportion of correct responses for the control, prefrontal, and thalamic lesion groups during Phase 4 of postsurgical training. Results are shown for each of the retention intervals, interposed from the offset of the tone to the raising of the gate, and for the choice point trials that were trained between blocks of rotation interval trials. 


\section{Phase $6 \mathrm{Go} /$ no go Performance}

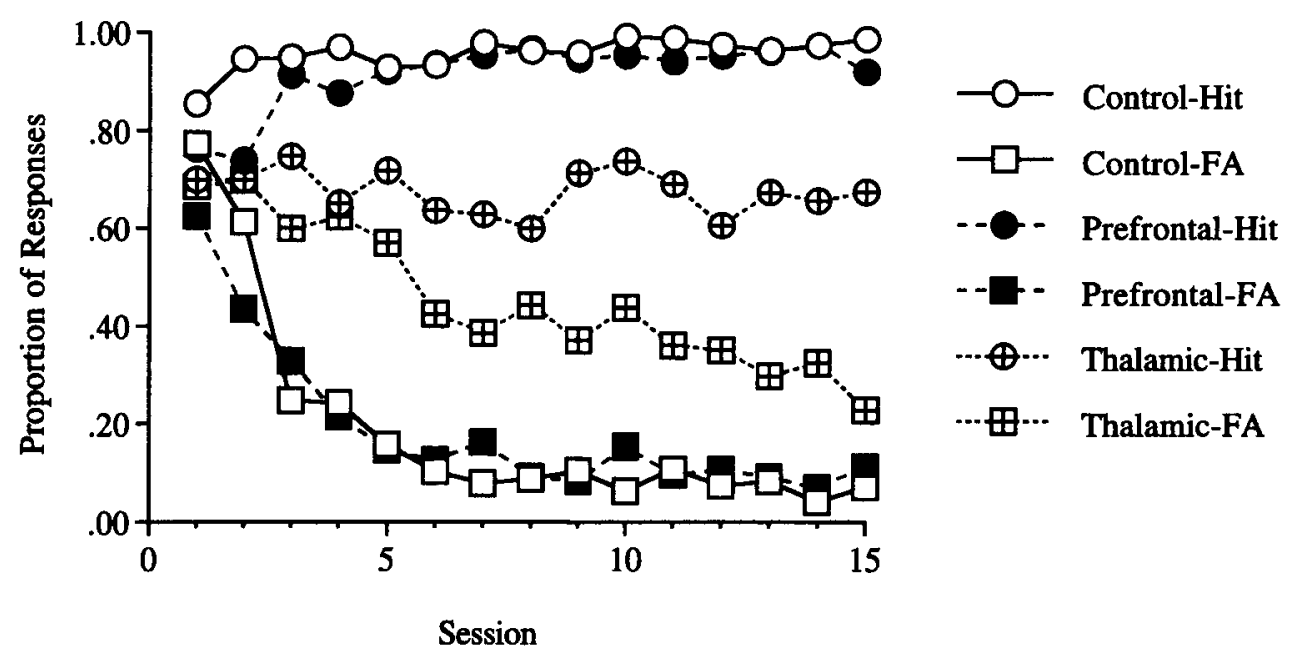

Figure 7. The proportion of correct responses on reinforced S+ trials (hit) and the proportion of incorrect responses on unreinforced $(S-$ ) trials (false alarm) of the go/no-go task. Results are shown for all 15 sessions for the control, prefrontal, and thalamic lesion groups.

thus, the rats needed only to associate the pitch of the positive stimulus with the availability of reinforcement and to extinguish responding to the negative stimulus through its association with nonreward. The selective impairment of AMTP seems consistent with several of the functions that have been linked to the PFC. These include proactive interference between competing response tendencies, the ability to learn conditional contingencies that involve distinct spatial or temporal events, and the capacity for inhibitory control of extraneous activity (Fuster, 1989; Milner \& Petrides, 1984; Robin \& Holyoak, 1995; Shimamura, 1995). Our data do not provide a critical test to decide between these alternative explanations.

The present findings are strikingly similar to a recent report that medial prefrontal lesions, comparable with the present $\mathrm{MW}$ lesions, disrupt the ability to perform a two-lever conditional discrimination while sparing the ability to perform a one-lever version of the same task (Winocur \& Eskes, 1998). Winocur (1991) first reported that lesions of the medial prefrontal cortex affect the ability of rats to perform a conditional discrimination task in which the location of a stimulus light indicates which of two adjacent retractable levers is associated with reinforcement on a given trial. In this study, prefrontal animals were impaired when the memory delay was minimized by extending the levers immediately after the light was turned off, and the extent of this impairment was independent of the length of the delay between the time when the light was turned off and the time when the levers were extended. In their more recent study, Winocur and Eskes replicated this result and demonstrated that the effects of the prefrontal lesion were greatly reduced when the rats were switched from the two-lever task to a one-lever task in which the requirement was essentially go (or press when the side illuminated corresponded to the side on which the lever extended)/no-go (or withhold when the sides did not correspond).

The differential effects of prefrontal lesions on the go/no-go and AMTP tasks in the present study and on the one- and two-lever tasks in Winocur and Eskes (1998) suggest that the PFC plays a critical role in conditional discriminations when rats are required to chose between two simultaneously available, spatially distinct response alternatives. These results are not surprising. They are consistent with the well-established effects of prefrontal lesions in rats on DMS, DNMS, and delayed alternation tasks in which two levers, alleys, or response ports serve as response alternatives (Eichenbaum, Clegg, \& Feeley, 1983; Harrison \& Mair, 1996; Kesner, Hunt, Williams, \& Long 1996; Larsen \& Divac, 1978; Porter \& Mair, 1997; van Haaren, de Bruin, Heinsbroek, \& van de Poll, 1985; Young et al., 1996). They are also consistent with the classic findings of delayed alternation and delayed response impairments in monkeys with prefrontal lesions (Fuster, 1989; Goldman-Rakic, 1987).

Although prefrontal lesions have been found to impair conditional discriminations between two spatially distinct response alternatives, other reports indicate that they have minimal effects on eight-arm radial maze tasks (once they have been acquired) in which rats are trained to retrieve one reinforcer from each alley without reentering alleys in which reinforcers have been consumed (Becker, Walker, \& Olton, 1980; Harrison \& Mair, 1996; Kolb, Pittman, Sutherland, \& Whishaw, 1982; Kolb, Sutherland, \& Whishaw, 1983; Porter \& Mair, 1997; Winocur \& Moskowitsch, 1990). These findings show that prefrontal lesions spare the ability to respond selectively to simultaneously available spatial locations when correct responding requires remembering information about recent arm entries. 
Porter and Mair (1997) have taken this analysis a step further by comparing performances of rats on several versions of two-choice DNMS trained in an automated eight-arm radial maze. Lesions of the PFC disrupted DNMS when rats had to choose between the same two arms on every trial (recurring-choice DNMS). Comparable results were observed for prefrontal lesions involving either the MW area or the MW area combined with cortical areas dorsal to the rhinal sulcus (i.e., all the cortex innervated by the MDn and, thus, defined by hodological criteria as prefrontal). By contrast, these same lesions had no effect on DNMS when the two choice arms were selected at random from the eight available options on a trial-by-trial basis (varying-choice DNMS). The ability to perform the varying-choice version of DNMS demonstrates a spared capacity to remember previous arm entries and to respond differentially to two arms on the basis of a DNMS contingency. The impairment observed on the recurring-arms version of DNMS suggests that repetition of response alternatives is an important factor determining the effects of prefrontal lesions. In other words, the PFC appears to be critical for conditional discriminations involving a simultaneous choice between two spatially distinct response alternatives when the same two alternatives are repeated on every trial.

The normal performance of the MW group on the auditory go/no-go task is consistent with other evidence that rats with this lesion can perform olfactory go/no-go DNMS and learn a go/no-go olfactory discrimination as well as controls can (Koger \& Mair, 1994; see also Otto $\&$ Eichenbaum, 1992). The auditory go/no-go task in the present study, like the go/no-go discriminations found to be insensitive to the effects of medial prefrontal lesions by Koger and Mair and Winocur and Eskes (1998), involved asymmetrical reinforcement in which positive stimuli were consistently associated with reinforcement and negative stimuli with nonreinforcement. With this reinforcement contingency, go/no-go discriminations require animals to learn to respond in the presence of the positive stimulus and to extinguish responding in the presence of the negative stimulus. The ability of prefrontal rats to perform asymmetrically reinforced go/no-go discriminations is consistent with the report of Petrides (1986) that, in monkeys, prefrontal periarcuate lesions spare the ability to perform auditory or visual go/no-go discriminations that are asymmetrically reinforced, while disrupting those that are symmetrically reinforced. With a symmetric contingency, reinforcements are associated with both the positive stimulus (for responding) and the negative stimulus (for withholding responding). Thus, correct performance requires that animals respond differentially to two stimuli that are both associated with reinforcement.

Investigators have related the effects of prefrontal cortical lesions to a number of psychological constructs. Much of this literature has focused on the role of the PFC in working memory or in the temporal organization of behavior (Fuster, 1989; Goldman-Rakic, 1987; Milner \&
Petrides, 1984). Although these constructs can account for a number of impairments associated with prefrontal lesions, they do not provide convincing accounts for the present results. First, AMTP was impaired during discrimination trials in which there was no temporal delay over which stimulus information had to be remembered before the discriminative response was made. Second, the AMTP deficit was not exacerbated when mnemonic demands were increased by imposing a temporal delay and increasing its length. Third, AMTP does not require rats to make a recency judgment of discriminative stimuli, as in DMS or DNMS tasks. Fourth, it is unclear how the concepts of working memory or of temporal organization can account for differential effects observed for the AMTP and the go/no-go discriminations or for the analogous findings reported by Winocur and Eskes (1998).

\section{Effects of Thalamic Lesions}

The rats with L-IML thalamic lesions performed poorly on both the AMTP and the go/no-go tasks. The impairment observed for AMTP is consistent with previous reports that L-IML lesions disrupt performance of delayed conditional discrimination tasks, including continuous olfactory DNMS (Koger \& Mair, 1994) or place DNMS or DMS (Mair \& Lacourse, 1992; Young et al., 1996). The impairment observed for AMTP provides evidence that these lesions affect conditional discrimination when there is no delay and no requirement to remember which discriminative stimulus was presented on a given trial.

The inability of the L-IML animals to perform the go/ no-go task was surprising. Previous studies have shown that these lesions spare the ability to learn and to perform discriminations in other sensory modalities, including olfactory go/no-go discrimination (Koger \& Mair, 1994) and place serial reversal learning (Harrison \& Mair, 1996). The impairments apparent for the auditory go/no-go task are compatible with at least two broad interpretations. First, the L-IML lesion may have more extensive effects on audition than on other sensory modalities. To test whether the impairment of the L-IML group reflected an inability to respond differentially to two pure tone stimuli, a second study was carried out to measure performance for AMTP and go/no-go discrimination, using a pure tone and white noise as discriminative stimuli (Stevens \& Mair, 1996). Simplifying the discrimination in this fashion had no effect on the impairment of L-IML animals on these tasks. Thus, if the inability of these animals to perform the go/no-go task is related to auditory dysfunction, it must involve something more basic than the capacity for pitch discrimination. It should also be noted that the L-IML lesion does not encroach upon the medial geniculate or any other known auditory relay nucleus. Second, the auditory go/no-go task may be more difficult than the place or olfactory discrimination tasks because of demands placed on attention, conditional learning, or some associated function not specifically related to audition. By this argument, L-IML rats are unable to 
meet task-specific demands of the auditory go/no-go procedure that are not related to the ability to respond differentially to the discriminative stimuli.

Although it is not clear why the L-IML group did so poorly, the inability of these animals to perform the go/ no-go tasks has at least two important implications for the present results. First, this failure raises questions about the validity of the AMTP data as a measure of mnemonic function of L-IML animals. If rats cannot respond reliably to differences between stimuli in a simple go/no-go discrimination, there would seem to be little reason to expect that they should be able to do so within the context of a delayed conditional discrimination. Second, the inability of the L-IML group to perform discriminations when there is no requirement that information be remembered across time must be taken as evidence that this lesion can affect basic aspects of sensory perception that do not involve memory. It is not yet certain whether comparable impairments can be demonstrated for other conditional discrimination tasks. Nevertheless, this possibility is consistent with evidence that L-IML lesions produce delay-independent impairments in conditional discrimination tasks, in which substantial deficits are apparent when the lengths of memory delays are minimized (Koger \& Mair, 1994; Mair \& Lacourse, 1992; Young et al., 1996).

The impairments observed for the L-IML rats in the present study may help to explain the neurological basis of perceptual impairments associated with human WernickeKorsakoff syndrome (WKS). Clinical evidence has established that human WKS is associated with medial thalamic lesions (Mair, 1994; Victor, Adams, \& Collins, 1989) and with deficits on perceptual tasks, extending to multiple sensory modalities, that place minimal demands on the capacity to remember information across time (Butters \& Cermak, 1980; Mair et al., 1986; Meudell, Mayes, MacDonald, Pickering, \& Fairbairn, 1991; Oscar-Berman, Goodglass, \& Cherlow, 1973; Oscar-Berman, Hutner, \& Bonner, 1992; Talland, 1965). For instance, Meudell et al. found that WKS patients were impaired in their ability to discriminate a sequence of two tones as high-low or low-high when presented at intertone intervals between 10 and $300 \mathrm{msec}$. The AMTP and go/no-go impairments observed for the L-IML group in the present study seem consistent with these types of perceptual impairment.

Although the present results do not provide a basis for differentiating between the contributions of the ILn and the MDn to the effects of L-IML lesions, there are several reasons to suspect the ILn may be particularly important. First, the more limited effects of the MW lesions on these tasks is inconsistent with the possibility that the MDn lesion is critical. If the MDn lesion were critical, it would seem likely that lesions in the MDn projections areas in the frontal cortex would produce deficits comparable with those from the L-IML lesion. It should also be noted that frontal cortical lesions in humans have not been associated with perceptual impairments comparable with those observed in human WKS patients (Damasio \& Anderson, 1993; Fuster, 1989). Second, other studies have shown that, in rats, lesions of the MDn that largely spare the adjacent ILn have little effect on DMS or DNMS tasks that use the locations of response ports or levers as discriminative stimuli (Burk \& Mair, 1998; Young et al., 1996). By contrast, lesions of the ILn have been associated with impairments of olfactory DNMS and place DMS comparable with those of L-IML lesions. Third, the anatomical and physiological properties of the ILn seem consistent with a critical role in processes related to attention or perception (see Groenewegen \& Berendse, 1994; Purpura \& Schiff, 1997).

Although experimental evidence is limited, the importance of the ILn for perception is supported by the results of a recent positron emission tomography study that showed signs of increased metabolic activity in the ILn when subjects were engaged in attention-demanding reaction time tasks involving stimuli in both visual and cutaneous modalities (Kinomura, Larson, Gulyas, \& Roland, 1996). Thus, the inability of the L-IML rats to perform the AMTP or go/no-go tasks seems consistent with perceptual impairments observed among human WKS patients (see particularly Meudell et al., 1991; Oscar-Berman et al., 1973; Oscar-Berman ei al., 1992) and, at least arguably, with what is known of the functional properties of the ILn.

\section{REFERENCES}

Aggleton, J. P., \& Mishkin, M. (1983a). Memory impairments following restricted medial thalamic lesions in monkeys. Experimental Brain Research, 52, 199-209.

Aggleton, J. P., \& Mishkin, M. (1983b). Visual recognition impairment following medial thalamic lesions in monkeys. Neuropsychologia, 21, 189-197.

Alvarez-Royo, P., Zola-Morgan, S., and SQuire, L. R. (1992). Impairment of long-term memory and sparing of short-term memory in monkeys with medial temporal lobe lesions: A response to Ringo. Behavioural Brain Research, 52, 1-5.

Becker, J. T., WALKer, J. A., \& Olton, D. S. (1980). Neuroanatomical bases of spatial memory. Brain Research, 200, 307-320.

BURK, J. A., \& MAIR, R. G. (1998). Thalamic amnesia reconsidered: Excitotoxic lesions of the intralaminar nuclei (ILn), but not the mediodorsal nucleus (MDn) disrupt place DMS performance. Behavioral Neuroscience, 112, 54-67.

ButTERS, N., \& CERMAK, L. S. (1980). Alcoholic Korsakoff's syndrome: An information-processing approach to amnesia. New York: Academic Press.

Damasio, A. R., \& Anderson, S. W. (1993). The frontal lobes. In K. M. Heilman \& E. Valenstein (Eds.), Clinical neuropsychology (pp. 409460). New York: Oxford University Press.

Eichenbaum, H., Clegg, R. A., \& Feeley, A. (1983). Reexamination of functional subdivisions of the rodent prefrontal cortex. Experimental Neurology, 79, 434-451.

Fuster, J. M. (1989). The prefrontal cortex. New York: Raven.

GolDMAN-RAKIC, P. S. (1987). Circuitry of primate prefrontal cortex and regulation of behavior by representational memory. In V. B. Mountcastle, F. Plum, \& S. R. Geiger (Eds.), Handbook of physiology: The nervous system (pp. 373-418). Bethesda, MD: American Physiological Society.

Groenewegen, H. J., \& Berendse, H. W. (1994). The specificity of the "nonspecific" midline and intralaminar thalamic nuclei. Trends in Neurosciences, 17, 52-57.

HARRISON, L. M., \& MAIR, R. G. (1996). A comparison of the effects of frontal cortical and thalamic lesions on measures of spatial learning and memory in the rat. Behavioural Brain Research, 75, 195-206.

Isseroff, A., Rosvold, H. E., Galkin T. W., \& Goldman-Rakic, P. S. (1982). Spatial memory impairments following damage to the mediodorsal nucleus in the thalamus of rhesus monkeys. Brain Research, 232, 97-113.

Kesner, R. P., Hunt, M. E., Williams, J. M., \& Long, J. M. (1996). 
Prefrontal cortex and working memory for spatial response, spatial location and visual object information in the rat. Cerebral Cortex, 6 , 311-318.

Kinomura, S., Larson, J., Gulyas, B., \& Roland, P. E. (1996). Activation by attention of the human reticular formation and thalamic intralaminar nuclei. Science, 271, 512-514.

KOGER, S. M., \& MAIR, R. G. (1994). A comparison of the effects of frontal cortical and thalamic lesions on measures of olfactory learning and memory in the rat. Behavioral Neuroscience, 108, 1088-1100.

Kolb, B., Pittman, K., Sutherland, R. J., \& Whishaw, I. Q. (1982). Dissociation of the contributions of the prefrontal cortex and dorsomedial thalamic nucleus to spatially guided behavior in the rat. Behavioural Brain Research, 6, 365-378.

Kolb, B., Sutherland, R. J., \& Whishaw, I. Q. (1983). A comparison of the contributions of the frontal and parietal association cortex to spatial localization in rats. Behavioral Neuroscience, 97, 13-27.

Kopelman, M. D. (1992). The "new" and the "old": Components of the anterograde and retrograde memory loss in Korsakoff and Alzheimer patients. In L. R. Squire \& N. Butters (Eds.), Neuropsychology of memory (2nd ed., pp. 130-146). New York: Guilford.

LaRSEN, J. K., \& Divac, I. (1978). Selective ablations within the prefrontal cortex of the rat and performance of delayed alternation. Physiological Psychology, 6, 15-17.

MAIR, R. G. (1994). On the role of thalamic pathology in diencephalic amnesia. Reviews in the Neurosciences, 5, 105-140.

Mair, R. G., Burk, J. A., \& Porter, M. C. (1998). In the rat (Rattus norvegicus) lesions of frontal cortex, hippocampus, and the intralaminar thalamic nuclei have distinct effects on remembering. $\mathrm{Be}$ havioral Neuroscience, 112, 772-792.

MaIR, R. G., Doty, R. L., Kelly, K. M., Wilson, C. S., Langlais, P. J., MCENTEE, W. J., \& VolLmecke, T. A. (1986). Multimodal sensory discrimination deficits in Korsakoff's psychosis. Neuropsychologia, 24, 831-839.

Mair, R. G., Ferguson, A. E., KNoth, R. L., \& Langlais, P. J. (1989). Widespread cortical denervation in rats following recovery from thiamine deficiency. Neuroscience Abstracts, 15, 1104.

MaIR, R. G., \& LACourSE, D. M. (1992). Radiofrequency lesions of thalamus produce delayed non-matching to sample impairments comparable to pyrithiamine-induced thiamin deficiency. Behavioral Neuroscience, 106, 634-645.

MAYES, A. R. (1992). What are the functional deficits that underlie amnesia? In L. R. Squire \& N. Butters (Eds.), Neuropsychology of memory (2nd ed., pp. 23-45). New York: Guilford.

Meudell, P. R., Mayes, A. R., MacDonald, C., Pickering, A., \& FAIRBAiRN, A. F. (1991). Korsakoff amnesics are poor at judging the sequence of two tones. Cortex, 27, 431-439.

Milner, B., \& PETRIDEs, M. (1984). Behavioural effects of frontal-lobe lesions in man. Trends in Neurosciences, 7, 403-407.

Mumby, D. G., Mana, M. J., Pinel, J. P. J., David, E., \& Banks, K. (1995). Pyrithiamine-induced thiamine deficiency impairs object recognition in rats. Behavioral Neuroscience, 109, 1209-1214.

Mumby, D. G., Pinel, J. P., \& Dastur, F. N. (1993). Mediodorsal thalamic lesions and object recognition in rats. Psychobiology, 21, 27-36.

Oscar-Berman, M., Goodglass, H., \& Cherlow, D. G. (1973). Perceptual laterality and iconic recognition of visual materials by Korsakoff patients and normal adults. Journal of Comparative \& Physiological Psychology, 82, 316-321.

OSCAR-Berman, M., Hutner, N., \& Bonner, R. T. (1992). Visual and auditory spatial and nonspatial delayed-response performance by Korsakoff and non-Korsakoff alcoholic and aging individuals. Behavioral Neuroscience, 106, 613-622.

Otro, T., \& Eichenbaum, H. (1992). Complementary roles of the orbital prefrontal cortex and the perirhinal-entorhinal cortices in an odor-guided delayed-non-matching-to-sample task. Behavioral Neuroscience, 106, 763-776.

PARKIn, A. J. (1987). Memory \& amnesia: An introduction. Oxford: Blackwell.

Paxinos, G., \& WATSON, C. (1986). The rat brain in stereotaxic coordinates (2nd ed.). New York: Academic Press.
PETRIDES, M. (1986). The effect of periarcuate lesions in the monkey on the performance of symmetrically and asymmetrically reinforced visual and auditory go, no go tasks. Journal of Neuroscience, 6, 2054-2063.

PorTer, M. C., \& MAIR, R. G. (1997). The effects of frontal cortical lesions on remembering depend on the procedural demands of tasks performed in the radial arm maze. Behavioural Brain Research, 87, 115-125.

PurPuRA, K. P., \& Schiff, N. D. (1997). The thalamic intralaminar nuclei: A role in visual awareness. Neuroscientist, 3, 314-321.

RoBIN, N., \& Holyoak, K. J. (1995). Relational complexity and the functions of prefrontal cortex. In M. S. Gazzaniga (Ed.), The cognitive neurosciences (pp. 987-997). Cambridge, MA: MIT Press.

RoBInson, J. K., \& MAIR, R. G. (1992). MK-801 prevents brain lesions and delayed non-matching to sample deficits produced by pyrithiamine-induced encephalopathy in rats. Behavioral Neuroscience, 106, 623-633.

Savage, L. M., \& Langlais, P. J. (1995). Differential outcomes attenuate memory impairments on matching-to-position following pyrithiamine-induced thiamine deficiency in rats. Psychobiology, 23, 153-160.

Shimamura, A. P. (1995). Memory and frontal lobe function. In M. S. Gazzaniga (Ed.), The cognitive neurosciences (pp. 803-813). Cambridge, MA: MIT Press.

SteVens, A. A., \& MAIR, R. G. (1995). Auditory delayed response task deficits following medial thalamic and prefrontal cortical lesions in the rat. Neuroscience Abstracts, 11, 1938.

Stevens, A. A., MaIR, R. G. (1996). [Auditory match-to-position: White noise vs. pure tone stimuli]. Unpublished raw data.

Talland, G. A. (1965). Deranged memory. New York: Academic Press.

TRAPOLD, M. A. (1970). Are expectancies based upon different positive reinforcing events discriminably different? Learning \& Motivation, 1, 129-140.

van Haaren, F., de Bruin, J. P. C., Heinsbroek, R. P. W., \& van de PoLL, N. E. (1985). Delayed spatial response alternation: Effects of delay-interval duration and lesions of the medial prefrontal cortex on response accuracy of male and female Wistar rats. Behavioural Brain Research, 18, 41-49.

Victor, M., Adams, R. D., \& Collins, G. H. (1989). The WernickeKorsakoff syndrome. Philadelphia: Davis.

WinOCUR, G. (1991). Functional dissociation of the hippocampus and prefrontal cortex in learning and memory. Psychobiology, 19, 11-20.

Winocur, G., \& Eskes, G. (1998). Prefrontal cortex and caudate nucleus in conditional associative learning: Dissociated effects of selective brain lesions in rats. Behavioral Neuroscience, 112, 89-101.

Winocur, G., \& Moskowitsch, M. (1990). Hippocampal and prefrontal cortex contribution to learning and memory: Analysis of lesion and aging effects on maze learning in rats. Behavioral Neuroscience, 104, 544-551.

Young, H. L., Stevens, A. A., Converse, E., \& Mair, R. G. (1996). A comparison of temporal decay in place memory tasks in rats with lesions of thalamus, frontal cortex, and the hippocampal system. $\mathrm{Be}$ havioral Neuroscience, 110, 1244-1261.

Zhang, Y., Burk, J. A., Glode, B. M., \& Mair, R. G. (1998). The effects of thalamic and olfactory cortical lesions on continuous olfactory DNMS and olfactory discrimination in the rat. Behavioral Neuroscience, 112, 39-53.

ZHANG, Y., \& MAIR, R. G. (1992). Denervation of frontal cortex in rats by RF lesions of the lateral internal medullary lamina (L-IML). Neuroscience Abstracts, 18, 520 .

ZILLES, K. (1985). The cortex of the rat: A stereotaxic atlas. Berlin: Springer-Verlag.

Zola-Morgan, S., \& SQuire, L. R. (1985). Amnesia in monkeys following lesions of the mediodorsal nucleus of the thalamus. Annals of Neurology, 17, 558-564.

(Manuscript received December 30, 1997; revision accepted for publication May 13, 1998.) 